\title{
Random lasing in an Anderson localizing optical fiber
}

\author{
Behnam Abaie ${ }^{1,2}$, Esmaeil Mobini ${ }^{1,2}$, Salman Karbasi ${ }^{3}$, Thomas Hawkins ${ }^{4}$, John Ballato ${ }^{4}$ and Arash Mafi,
}

A directional random laser mediated by transverse Anderson localization in a disordered glass optical fiber is reported. Previous demonstrations of random lasers have found limited applications because of their multi-directionality and chaotic fluctuations in the laser emission. The random laser presented in this paper operates in the Anderson localization regime. The disorder induced localized states form isolated local channels that make the output laser beam highly directional and stabilize its spectrum. The strong transverse disorder and longitudinal invariance result in isolated lasing modes with negligible interaction with their surroundings, traveling back and forth in a Fabry-Perot cavity formed by the air-fiber interfaces. It is shown that if a localized input pump is scanned across the disordered fiber input facet, the output laser signal follows the transverse position of the pump. Moreover, a uniformly distributed pump across the input facet of the disordered fiber generates a laser signal with very low spatial coherence that can be of practical importance in many optical platforms including image transport with fiber bundles.

Light: Science \& Applications (2017) 6, e17041; doi:10.1038/lsa.2017.41; published online 25 August 2017

Keywords: disordered optical fiber; random lasing; transverse Anderson localization

\section{INTRODUCTION}

Unlike conventional lasers that require a resonator cavity to operate, random lasers exploit multiple scattering to trap light and provide feedback to the system ${ }^{1-3}$. One of the first observations of random lasing was emissions from a laser dye solution containing microparticles ${ }^{4-6}$. Since then, there have been several reports that attribute random lasing in disordered media either to diffusive extended modes or Anderson localized modes ${ }^{2,7-13}$. Anderson localization was first used by Pradhan and Kumar ${ }^{14}$ to show increased reflected intensity due to wave confinement in a mirror-less amplifying one-dimensional (1D) structure. Later, enhancement of lasing in random multilayer stacks and planar waveguides was explained conceptually by Anderson localization ${ }^{15,16}$. Notably, the presence of narrow spikes in the emission spectrum of a semiconductor powder zinc oxide was associated with the formation of Anderson localized modes ${ }^{8}$. However, further experiments showed that the narrow spikes of a random laser can be observed in nearly the entire range of scattering strengths, even for cases where it is unlikely for Anderson localization to play a role ${ }^{17}$. Later, statistical analysis of passive mode decay rates in disordered media revealed the importance of long-lived extended modes in the presence of gain and numerical calculations showed that they can form lasing modes with narrow emission spectra ${ }^{18-20}$.

The nature of lasing modes in disordered media, particularly the role of Anderson localization in these systems, is still a matter of debate $^{21-23}$. Currently, it is accepted that Anderson localization is not required for coherent random lasing in disordered media ${ }^{24,25}$. However, Anderson localized lasing modes can result in a narrower frequency response analogous to closed cavities in regular lasers ${ }^{26}$.
Here we present a disordered laser system for which Anderson localization plays an integral role in determining its lasing characteristics - the outcome is the observation of spectral narrowing attributed to localized-mode quasi-cavities. The flexible glass optical fiber-based system used here allows the coverage of a broad range of parameters that set the system in configurations ranging from non-Andersonlocalized to Anderson-localized lasing regimes, hence enabling the strict attribution to Anderson localization-based lasing.

Observation of Anderson localization in three-dimensional optical systems is quite challenging ${ }^{27-29}$ because the refractive index contrast achievable with low-loss optical materials is too small to satisfy the Ioffe-Regel criterion ${ }^{30}$. However, unbounded one- and twodimensional disordered systems are always Anderson localized-for the bounded systems is sufficient to ensure that the system dimensions are substantially larger than the localization length to minimize the impact of boundary on localization ${ }^{31-38}$. As such, a transversely disordered and longitudinally invariant optical structure provides a quasi-one-dimensional (quasi-1D) or quasi-2D random system that can evade the Ioffe-Regel criterion. In such a medium, an optical wave can propagate freely in the longitudinal direction and remain localized in the disordered transverse dimension(s). This phenomenon is called transverse Anderson localization (TAL) ${ }^{39-42}$. In 2012, Karbasi et al. ${ }^{43-46}$, reported the first Anderson localizing optical fiber fabricated by polymer and glass (g-ALOF) ${ }^{47}$. The glass fiber reported in Ref. 47 has a randomly distributed air-hole pattern in transverse dimensions which remains invariant along the length of the fiber for the typical lengths used in Ref. 47 and here. The air fill fraction was shown to be higher near the outer boundaries and therefore localization is stronger

${ }^{1}$ Department of Physics and Astronomy, University of New Mexico, Albuquerque, NM 87131, USA; ${ }^{2}$ Center for High Technology Materials, University of New Mexico, Albuquerque, NM 87106, USA; ${ }^{3}$ Department of Electrical and Computer Engineering, University of California, San Diego, CA 92093, USA and ${ }^{4}$ Center for Optical Materials Science and Engineering Technologies (COMSET) and the Department of Materials Science and Engineering, Clemson University, Clemson, SC 29625, USA

Correspondence: A Mafi, Email: mafi@unm.edu

Received 9 November 2016; revised 6 March 2017; accepted 8 March 2017; accepted article preview online 10 March 2017 
near the boundaries in comparison to the central regions of the fiber. For more details about the disorder structure of g-ALOF please see Supplementary Fig. S4.

The air-holes of g-ALOF are filled with an active material using capillary action $^{48-50}$. This provides an opportunity to investigate lasing features in such a transversely random and longitudinally invariant structure. Here we report the first observation of directional random lasing in a g-ALOF. The strong transversely localized modes of the passive fiber provide the basis for Anderson localized lasing modes: the lasing modes in g-ALOF highly resemble the modes of the passive fiber where localization is stronger near the outer boundaries in comparison to the central regions of the fiber. We explore the spectral and temporal behavior of this laser configuration. The spectral narrowing dictated by the Anderson-localized modes of the system is clearly observed. In the temporal domain, pulse shortening beyond the lasing threshold is observed as expected and reported in the past ${ }^{4,6}$.

\section{MATERIALS AND METHODS}

\section{Fabrication of the fiber}

g-ALOF used in this work is drawn from 'satin quartz' (Heraeus Quartz), which is a porous artisan glass. The initial rod is $8 \mathrm{~mm}$ in diameter and $850 \mathrm{~mm}$ in length and is drawn at Clemson University on a Heathway draw tower at a temperature of $1890^{\circ} \mathrm{C}$. The tip of the fiber is imaged using a Hitachi SU-6600 analytical variable pressure field emission scanning electron microscope (SEM). The diameter of the fiber is $\sim 250 \mu \mathrm{m}$ and the average air fill-fraction is about $5.5 \%$ with the air-hole diameters varying between about 0.2 and $5.5 \mu \mathrm{m}$.

\section{Active fiber preparation and measurements}

The outer acrylate coating of g-ALOF is removed using a hot air gun. A piece of g-ALOF with $\sim 10 \mathrm{~cm}$ length is cleaved by a York PK Technology fiber cleaver and dipped inside a rhodmaine 640 solution in ethanol or benzyl alcohol (or a mixture of both) at a concentration of $0.5 \mathrm{mg} \mathrm{ml}^{-1}$ for about $6 \mathrm{~h}$. The tip of the dye-filled g-ALOF is investigated under a microscope to ensure the holes are uniformly filled with the dye solution. A $10 \mathrm{~mm}$ piece of the dye-filled g-ALOF is cleaved and mounted on an adjustable fiber clamp to be used in the experiments. The dye-filled g-ALOF is end-pumped by a frequency doubled Nd:YAG laser with a pulse duration of $0.6 \mathrm{~ns}$ and a repetition rate of $50 \mathrm{~Hz}$, using a $\times 20$ microscope objective (OBJ1 in Supplementary Fig. S5). The repetition rate and pump energy is kept low to delay the optical bleaching of the dye. The near-field image at the output tip of active g-ALOF is imaged on a CCD beam profiler by a $\times 40$ microscope objective (OBJ2 in Supplementary Fig. S5).

\section{Numerical simulations}

The guided modes of g-ALOF are calculated using COMSOL Multiphysics ${ }^{51}$. The Floating Network Licensed software is installed on a cluster located at the Center for Advanced Research Computing of the University of New Mexico, meeting large memory requirements for the heavy calculations. The geometry of g-ALOF is imported to COMSOL using the SEM image of the tip of the fiber by rendering the image into a compatible vector format (DXF) using Inkscape Vector Graphics Editor software. The refractive index profile of g-ALOF, used in the simulations, is shown in Supplementary Fig. S6.

\section{Characterization of the spectral stability}

In order to characterize the spectral stability of the laser in the Anderson localized regime, the spectrum is measured in a sequential mode such that 100 data acquisitions are done in $3 \mathrm{~s}$ (each spectrum is integrated over $30 \mathrm{~ms}$ ). Such a fast data acquisition provides the
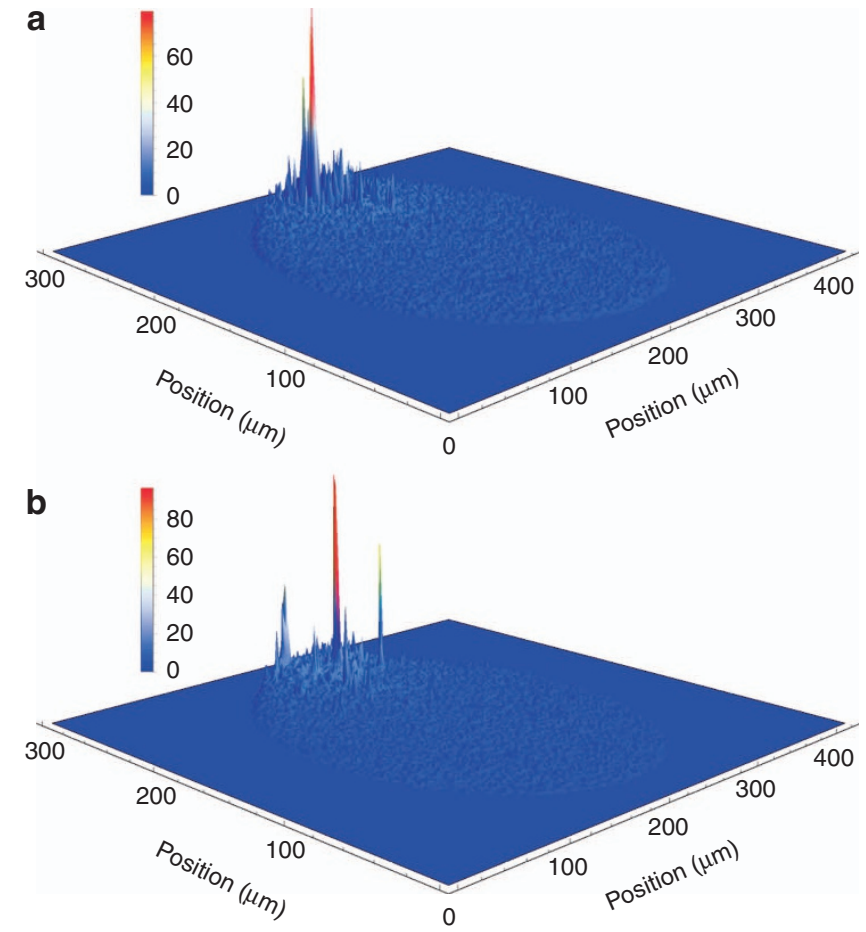

Figure 1 TAL of pump and laser in the mixed regime. Anderson localization of the: (a) pump beam near the edge, and (b) laser beam associated with the pump condition in a. Narrow peaks in the laser beam profile is due to the enhancement of lasing mediated by step-index guiding inside the individual holes of the active g-ALOF.

possibility to compare the laser emission spectra under excitations with individual shots of a pump pulse train with $50 \mathrm{~Hz}$ repetition rate. In order to quantify the fluctuations of the laser spectrum under excitations with individual pump pulses, we use the normalized mean integrated squared error (NMISE) defined by

$$
\text { NMISE }=\frac{\sum_{i}^{N} \int\left(f_{i}(\lambda)-\bar{f}(\lambda)\right)^{2} d \lambda}{N \times \int(\bar{f}(\lambda))^{2} d \lambda} \times 100,
$$

where $f_{1}(\lambda), f_{2}(\lambda), \ldots, f_{N}(\lambda)$ are the data collected for the laser spectrum at $N$ successive identical pump pulses, and $\bar{f}(\lambda)$ is the average of them. NMISE ranges between 0 and $100 \%$, where $0 \%$ is proportional to a series of completely identical spectra and $100 \%$ is related to a series of completely distinct spectra.

\section{RESULTS AND DISCUSSION}

Transversely localized lasing

The pump and laser beam profiles at the output facet of g-ALOF are shown in Figure 1. The pump beam at the input facet is coupled near the edge of g-ALOF. The residual pump in the output remains clamped in the same transverse location, consistent with Anderson localization of g-ALOF presented in Ref. 47. The laser beam profile, shown in Figure 1b, is also localized at the same transverse position. Note that in these results, the dye (benzyl solution) has a higher refractive index than the host glass; therefore, step-index guiding is playing a role for individual dye filled air-holes of g-ALOF. The stimulated emission is enhanced in these regions due to a stronger interaction of laser beam and gain; therefore, step-index guiding manifests itself in the form of strong discrete peaks in the laser beam 

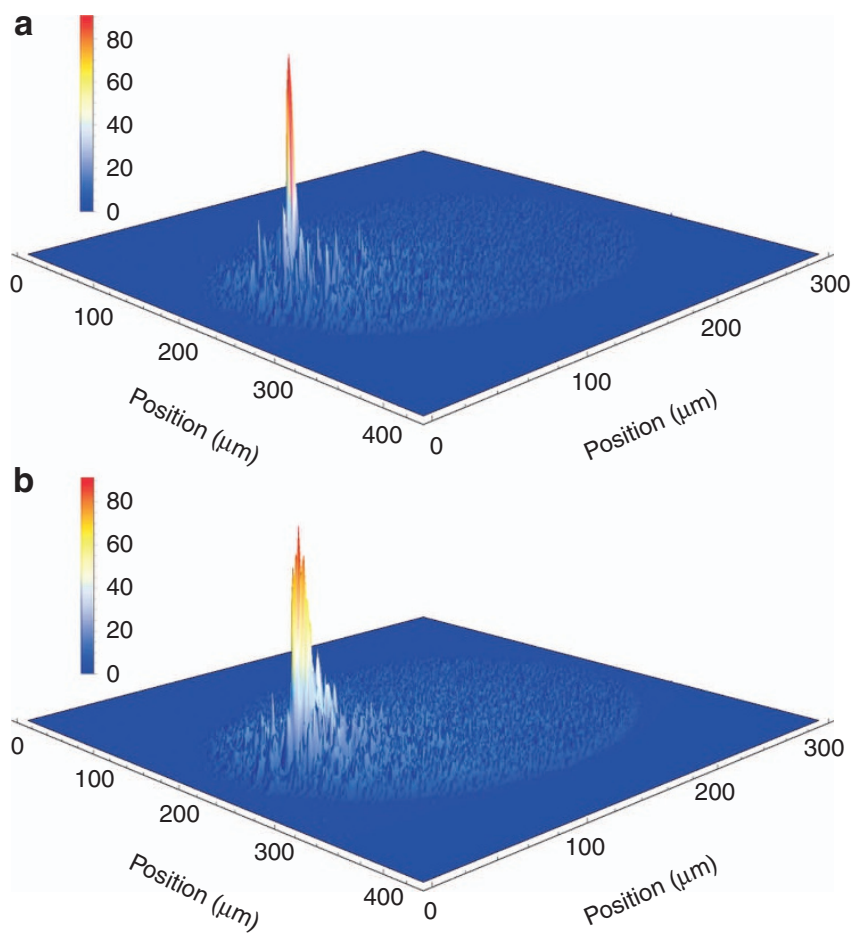

Figure 2 TAL of pump and laser in the Anderson-localized regime. Anderson localization of the: (a) pump beam near the edge, and (b) laser beam associated with the pump condition in a. The air-holes of g-ALOF are filled with rhodmaine 640 solution in ethanol; therefore, step-index guiding is not playing a role. Laser beam profile is transversely localized and follows the transverse position of the pump.

profile in Figure 1b. Nevertheless, TAL is present at least in the form of a localized pump beam. Because the hallmarks of both TAL and step-index guiding are simultaneously present in these results, this regime of laser operation is referred to as the 'mixed regime'. In the following section, results that are solely due to TAL are presented for which the step-index guiding is completely absent and the spatial behavior of the laser beam is entirely dictated by Anderson localization.

In pursuance of evading step-index guiding, the air-holes are filled with rhodmaine 640 solution in ethanol. The refractive index of ethanol (1.37) is smaller than that of the host glass (1.46); therefore, individual air holes filled with the solution do not form local waveguides in the active medium. Figure 2 shows the transverse localization of the pump and laser near the edge of g-ALOF filled with this solution. This behavior is fully dictated by the TAL of the pump and laser: in the absence of TAL, the focused pump beam coupled near the edge of g-ALOF would have quickly diffracted and covered the entire cross section of g-ALOF, given that the Rayleigh range $Z_{R}$ for the focused pump is on the order of $Z_{\mathrm{R}} \sim 200 \mu \mathrm{m}$. Figure 2a shows that TAL clearly dominates diffraction mediated by the strong transverse disorder in g-ALOF and confines the pump beam around its incoming transverse position as it freely propagates along the fiber. Notice that, based on Ref. 47, the effective beam radius of the pump expands as the beam propagates along the fiber until it reaches its final localized value, after which it does not change substantially. Numerically it was shown that the stabilized effective beam radius is reached after about $30 \mathrm{~mm}$ of propagation in g-ALOF.
The active g-ALOF used here is $10 \mathrm{~mm}$ long and the scattering strength is also reduced due to the dye filling, effectively leading to a longer stabilization distance. Therefore, the effective beam radius of the residual pump at the output facet of g-ALOF is not fully stabilized. Nevertheless, it is quite close to the stabilized value ${ }^{47}$. The laser beam profile associated with the pump condition described above is shown in Figure 2b. It is transversely localized and highly resembles the pump beam profile. However, the laser effective beam radius is at its stabilized form because of the laser stabilization condition that requires multiple round trips and amplification in the cavity. Notice that the localized laser beam in an active g-ALOF requires a localized pump beam, because g-ALOF supports numerous localized modes that are distributed across the transverse dimensions of the fiber and an extended non-localized pumping can excite many of these localized modes simultaneously, resulting in an extended output laser signal. We emphasize that in a non-disordered fiber without mode localization, even localized pumping does not result in localized lasing.

In Figures 1 and 2, the output laser beam follows the transverse position of the input pump. Localized states in g-ALOF trap the beam, which is propagating back and forth between air-fiber interfaces. In other words, the disorder induced localized states form several isolated channels located across the transverse dimension of g-ALOF. On excitation of one of these channels by a narrow input pump, the system starts lasing by the feedback provided through the $4 \%$ reflection at each air-fiber interface. Therefore, the presence of the TAL and the Fabry-Perot formed by the air-fiber interfaces results in a directional random laser. Evaluation of g-ALOF laser beam quality based on the variance method ${ }^{52,53}$ followed by a short discussion about the directionality is provided in Supplementary Information.

As we mentioned earlier, TAL is stronger near the boundaries of g-ALOF in comparison to the central regions of the fiber due to the higher disorder in these regions ${ }^{47}$. In order to compare the results with previous analysis on passive g-ALOF, the pump beam is launched at two different transverse positions of the active g-ALOF by scanning the pump objective using a precision XYZ translation stage. Figure $3 \mathrm{a}$ and $3 \mathrm{~b}$ show the laser beam profile at the output facet of g-ALOF when the input pump beam is launched near the edge and the center of g-ALOF input facet, respectively (residual pump beam profiles are not exhibited in Figure 3). Clearly, Anderson localization occurs more strongly around the edge of g-ALOF in agreement with the results reported in Ref. 47. Because the presented results are solely due to TAL and step-index guiding is absent, we call this regime of laser operation as 'Anderson-localized regime' in the sections that follow.

\section{Origin of localized lasing}

Localized lasing modes. In order to further investigate the presence of localized lasing modes in active g-ALOF, we have numerically calculated guided modes of the system using the Finite Element Method. A small imaginary part is added to the refractive index of the dye-filled air-holes to represent the gain. The refractive index profile used for simulations is shown in Supplementary Fig. S6. If calculated modes are Anderson localized, the system can potentially support localized lasing. Here we only show results in the Anderson-localized regime of laser operation, but similar results have been verified for the mixed regime as well.

The simulations are performed in the frequency domain for a wavelength that falls inside the emission spectrum of the laser (around $610 \mathrm{~nm}$ ). The refractive index of the host glass is set at 1.46, and that of the dye-filled air-holes is set at 1.37 with a small imaginary part. Figure 4a shows the time-averaged power flow of a typical calculated mode. The background pattern is the transverse geometry of g-ALOF 

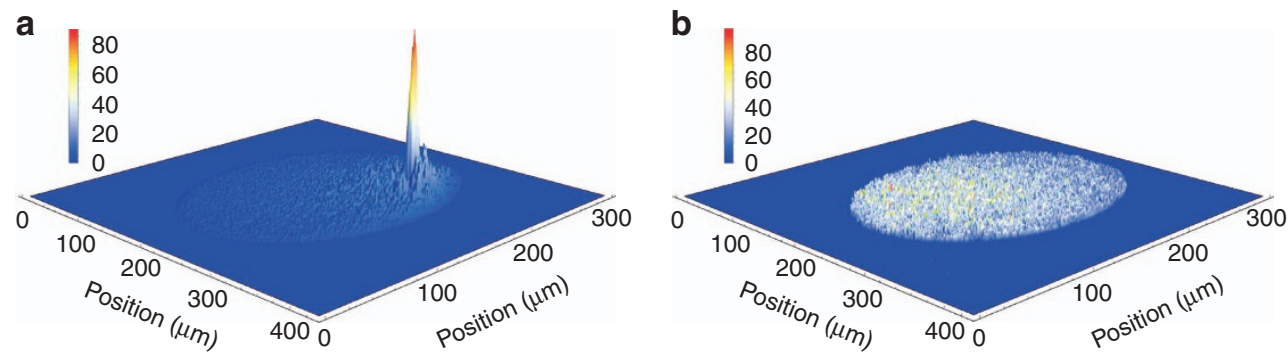

Figure 3 Stronger localized lasing near the edge of g-ALOF. Localization of the laser is stronger near the boundaries. A narrow pump beam is scanned across the input facet of g-ALOF to excite disorder-induced channels (a) near the edge, and (b) at the center of the disordered fiber, respectively. Stronger TAL near the edge results in a narrower output laser in comparison to the central regions.

a

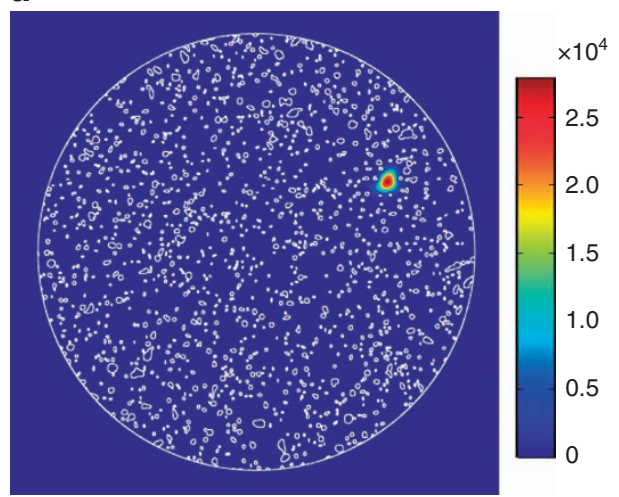

b

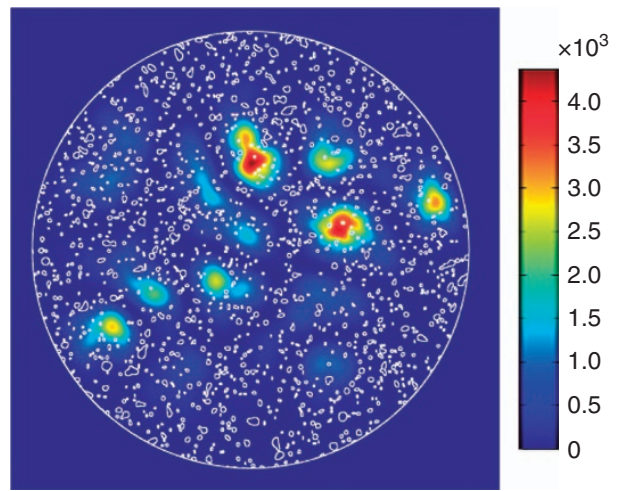

Figure 4 Calculated localized lasing modes. Time average power of typical calculated lasing modes. (a) The localization length is smaller than the system size due to strong transverse disorder and TAL. (b) Reduced scattering strength, in comparison to a, leads to a weaker TAL, and therefore localization length is larger than the system size and modes nearly cover the entire transverse dimensions of g-ALOF.

extracted directly from its SEM image. The calculated mode is clearly localized due to the presence of transverse disorder and strong multiple scattering. We note that other methods such as local stepindex guiding or photonic bandgap guiding can also be used to localize light but are not playing a role in the structure studied here ${ }^{42,47}$. The impact of TAL can be verified by decreasing the refractive index contrast between the dye-filled air-holes and the host glass. In this regard, Figure $4 \mathrm{~b}$ shows a typical guided mode with exactly the same configuration as Figure 4 a except with the refractive index of the dye-filled air-holes set at 1.459. Decreasing the refractive index contrast and therefore scattering strength has clearly reduced the impact of TAL such that the localization radius of the mode is larger than the system size, so it appears to be nearly extended over the entire transverse dimensions of g-ALOF. More examples of localized lasing modes are presented in Supplementary Fig. S7.

Localization in passive g-ALOF. TAL in g-ALOF has been rigorously explored in Ref. 47. However, the reported results were for a passive g-ALOF without any dye filling. It is important to note that filling the air holes of g-ALOF with ethanol or benzyl alcohol reduces their refractive index contrast with the host glass which causes a weaker $\mathrm{TAL}^{44}$. Another important difference is in the wavelengths of the laser and pump used here in comparison to the $405 \mathrm{~nm}$ diode laser used in Ref. 47. Here we report TAL in g-ALOF filled with ethanol. Results are similar to the case of g-ALOF filled with benzyl alcohol, which are not reported here. We emphasize that the ethanol used here is pure ( $99 \%$ purity) with no dye solute. A $\mathrm{He}-\mathrm{Ne}$ laser (center wavelength $\sim 633 \mathrm{~nm}$ ) is used to carry out the experiments, because its wavelength is in the emission range of g-ALOF laser that is reported later in this paper (Figure 6).

The experimental setup is similar to Supplementary Fig. S5 except that the Nd:YAG laser is replaced with the He-Ne laser, and g-ALOF used in the experiment is filled with pure ethanol. Figure 5 shows near-field image of the tip of g-ALOF at the output. The input objective (OBJ1 in Supplementary Fig. S5) is scanned across the input facet of g-ALOF to compare localization near the boundaries with the central regions. Clearly, localization is stronger when the objective launches the input beam near the boundary of g-ALOF, shown in Figure 5a, compared with Figure 5b, where the input beam is launched near the center. These results show that g-ALOF supports strongly localized modes in the spectral emission range of g-ALOF laser even when the air holes are filled with ethanol, in comparison with Ref. 47 where the air holes are open.

\section{Laser spectrum}

Anderson-localized regime. In Ref. 54, lasing in a dye filled porous glass disk was reported. The scattering strength in the system was weak and therefore the results were in the diffusive regime. It was shown that, the emission spectra of such an amplifying disordered medium were distinct and uncorrelated at each shot of the identical pump pulses. This behavior was explained based on the strong coupling of modes initiated by spontaneous emission, and Anderson-localized regime was suggested to reduce mode competition and chaotic behavior of the random laser. Here we study the stability of g-ALOF 
a

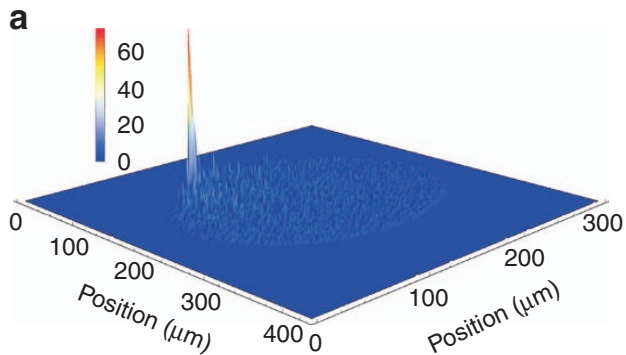

b

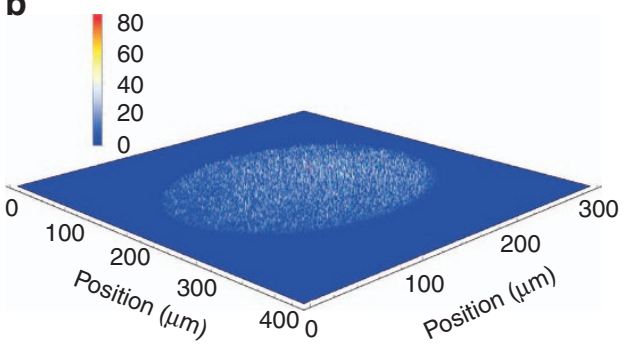

Figure 5 Localization in passive g-ALOF. TAL in passive g-ALOF filled with ethanol: (a) near the edge, and (b) at the center. The disordered fiber supports strongly localized modes in the spectral emission range of g-ALOF laser even when the air holes are filled with ethanol, in comparison with Ref. 47 where the air holes are open. TAL is stronger near the boundary of g-ALOF in agreement with Ref. 47.
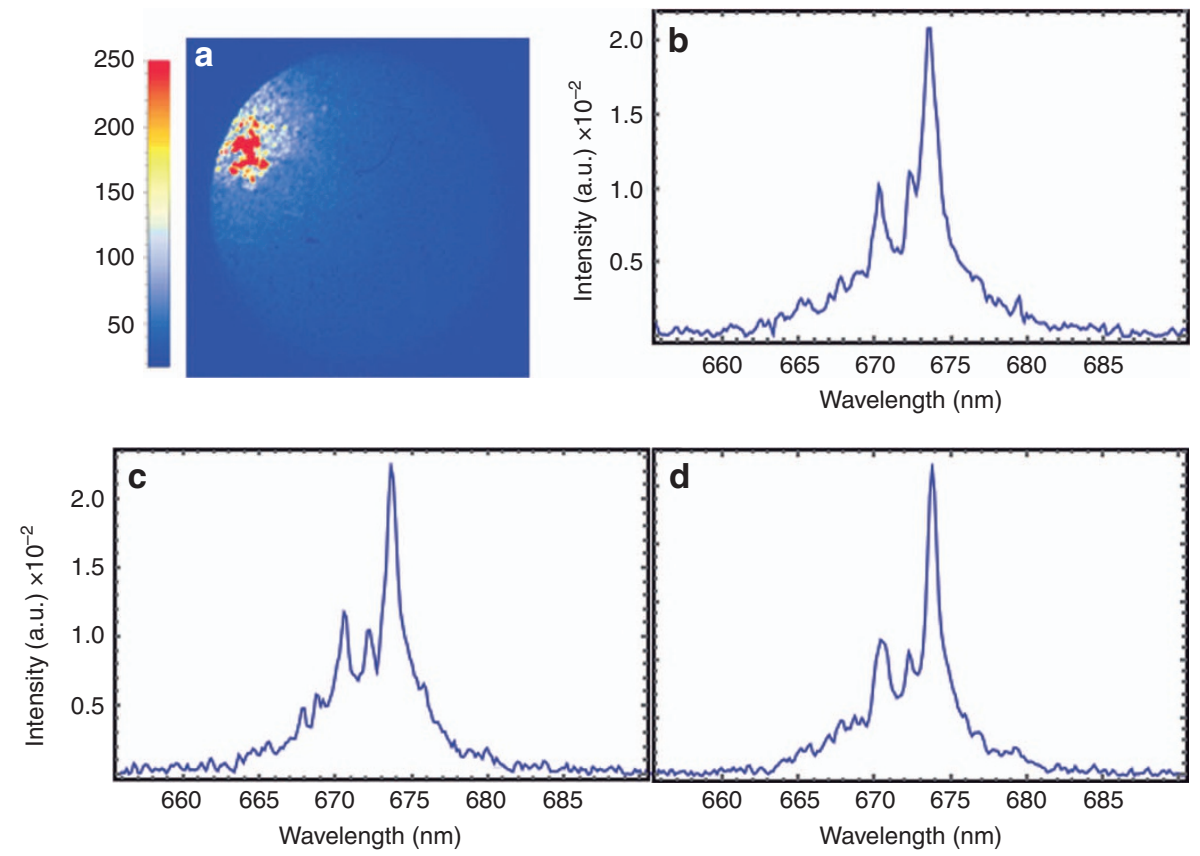

Figure 6 Laser emission spectrum in the Anderson localized regime. (a) A strongly localized lasing mode and its associated spectra under excitation with three individual pump pulses in (b-d). The spectrum shows high stability as the narrow spikes remain the same under excitation with individual pump pulses in contrast to the results presented in Ref. 54 .

laser emission spectra. Our results show that, for a strongly localized lasing mode, the spectrum remains nearly unchanged at consecutive shots of the pump pulse train.

Figure 6a shows a strongly localized lasing mode and the associated emission spectra; the emission spectrum under pumping with three successive identical pulses are shown in Figure $6 \mathrm{~b}-6 \mathrm{~d}$. The spectrum shows a relatively high stability; the narrow spikes remain at the same wavelength in contrast to Ref. 54 where the spikes appeared at distinct wavelengths under excitation with identical pump pulses. In order to quantify the stability of g-ALOF laser spectrum, we use NMISE (see Materials and Methods), as a measure of the spectrum fluctuations over 100 successive identical pump pulses. The calculated value of NMISE for the strongly localized mode in Figure $6 a$ is very small $(\sim 4 \%)$, which indicates the high spectral stability. Further information about the dependency of the spectral stability to the localization strength of the lasing modes is presented in Supplementary Information.

High spectral stability of g-ALOF laser is understood based on the strong mode confinement granted by the localized states; as discussed earlier, disorder induced localized states form guiding channels located across the transverse dimension of the disordered fiber. Once a narrow pump beam excites one of these channels, the system starts lasing by the feedback provided through reflections at air-fiber interfaces. TAL reduces mode competition which is the underlying mechanism that causes chaotic fluctuations in the emission spectra of random lasers with weak mode confinement such as the one reported in Ref. 54 .

Mixed regime. Figure 7 shows the emission spectrum in the mixed regime at different pump pulse energy levels. Inset shows the linewidth narrowing with respect to the pump energy. As expected, the laser spectrum narrows as the pump power increases; narrow spikes on top of the global narrowing of the laser spectrum are evident. In this regime of laser operation, the emission spectrum depends highly on the pump condition, where scanning the pump across the fiber input facet affects the spectrum significantly (not shown here). This is due to the local waveguides formed at various transverse positions of the fiber as explained previously. Because these local waveguides have diverse geometries due to the random nature of the fiber, emission spectrum 


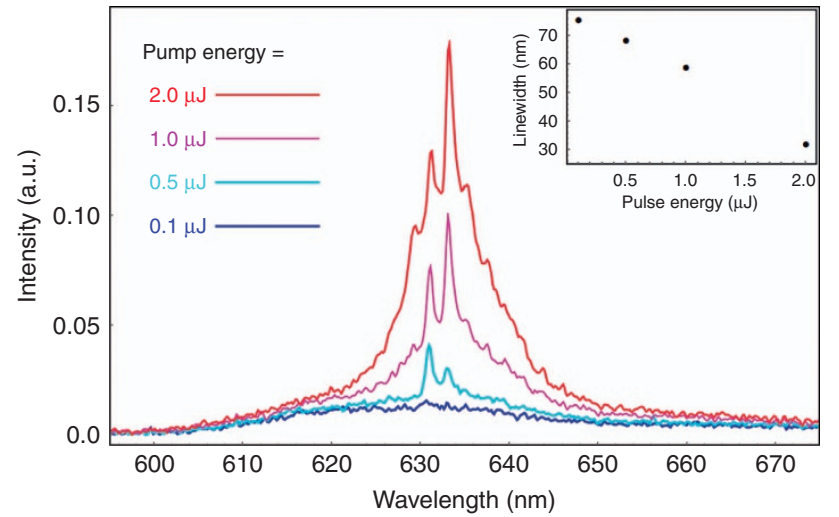

Figure 7 Laser emission spectrum in mixed regime. Narrow spikes on top a global narrowing of the spectrum can be seen. Emission spectrum in this regime of laser operation is highly sensitive to the pump position due to the impact of step-index guiding inside local waveguides with diverse geometries across the disordered fiber. Inset shows the linewidth narrowing with respect to the pump energy.

varies as the pump is scanned, exciting different local waveguides across the fiber cross-section. We note that we have also analyzed the dependence of the lasing wavelength on the dye material and its concentration, but the results are not presented here because they were consistent with frequently reported characteristic signatures of the dye lasers ${ }^{48,49}$. In all results of this section, we have used the same dye material with equal concentration to avoid their impact on our analysis.

\section{Pulse shape}

The laser signal is measured in the time domain using a $<300$ ps response-time photodetector and an $8 \mathrm{GHz}$ oscilloscope for various pump energies below and above lasing threshold. Figure $8 \mathrm{a}$ shows the laser pulse in Anderson-localized regime and Figure $8 \mathrm{~b}$ shows it in the mixed regime. The insets in both figures show the temporal profile of the pump pulse. When the pump pulse temporal front (left side) hits the dye molecules the signal rapidly grows as can be seen in the temporal front (left side) of the signal. Well below the lasing threshold ( $1.4 \mu \mathrm{J}$ pump pulse energy in Figure 8a), the excited dye molecules go through a (relatively slow) spontaneous decay, which explains the slow fall-off in the temporal back (right side) of the spontaneous emission signal. The exponential decay in the pulse tail has a $\sim 5 \mathrm{~ns}$ time constant in agreement with the previously reported emission lifetime of rhodamine $640^{55}$. In the opposite case when the pump energy is high enough to set the system well above the lasing threshold $(10 \mu \mathrm{J}$ pump pulse energy in Figure 8a), the laser pulse follows almost exactly the temporal profile of the pump as is expected when the stimulated emission dominates the spontaneous emission in the laser dynamics. The case with $4.5 \mu \mathrm{J}$ pump pulse energy in Figure 8a represents the transition region where there is a mixture of stimulated and spontaneous emission. Similar behavior is observed in the mixed regime shown in Figure 8b. However, the threshold is quite smaller in the mixed regime because of the larger interaction between the gain medium and the optical field due to step-index wave-guiding. The strong interaction results in a larger effective gain hence lowering the lasing threshold.

The reported observations clearly indicate the underlying role of Anderson localization in the lasing behavior of the random fiber laser. The modes of this laser are transversely localized and strongly resemble the modes of the passive system. The dynamics of lasing is
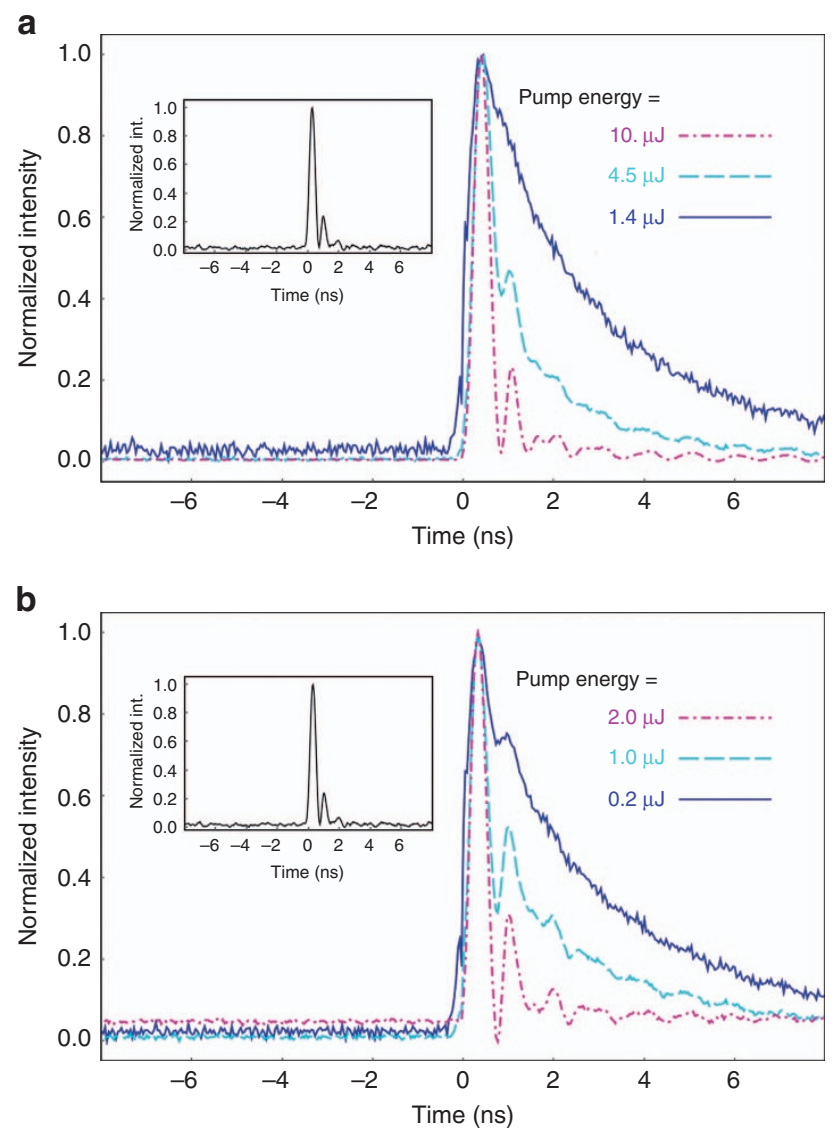

Figure 8 Laser pulse shape. Laser pulse in (a) Anderson-localized regime, and (b) mixed regime of laser operation where the inset is related to the pump pulse. Pulse shortening beyond threshold is apparent in both regimes of laser operation.

dictated by the coupling between the transverse quasi-cavities formed by Anderson-localized modes and the longitudinal Fabry-Perot cavity established by the $4 \%$ reflections at the air-fiber interface of fiber tips. The key observation is directional random lasing mediated by Anderson localization in an optical fiber medium.

Applications of previously reported random lasers are limited mainly because of their multi-directional emission and chaotic fluctuations, where their weak mode confinement results in a high degree of mode competition and therefore chaotic fluctuations. The demonstrated flexible fiber based random laser in this work clearly operates in the regime of Anderson localization leading to a spectrally stable and highly directional random laser. Disorder induced localized states form several isolated channels located across the transverse dimension of g-ALOF. The presence of these guiding channels in conjunction with the Fabry-Perot formed between g-ALOF tips assures a directional random laser. In this implementation, if a narrow pump beam excites one of the disorder induced channels across g-ALOF input facet, the output laser beam follows the transverse position of the pump. On the other hand, under extended input pumping g-ALOF laser results in a laser beam with a very low spatial coherence; we investigate and verify this in the Supplementary by performing the Young's double-slit experiment ${ }^{56,57}$.

Recently, random lasers have been applied for speckle-free imaging, where it is shown that spatial incoherency helps in avoiding the formation of speckle patterns in optically rough media ${ }^{58}$. In Ref. 46, the randomness in a disordered fiber was used to scramble the 
incoming spatially coherent laser light and ensured an effective spatial incoherence that improved the image transport metrics. We suggest that using a coherent fiber bundle but with a spatially incoherent laser beam can achieve the same goal, constituting a viable practical application for the system studied in this paper.

The presented work should be viewed as a proof of concept and a proposed platform for potential practical applications. We note that dye-based lasers suffer from optical bleaching; however, it is possible to circulate a dye solution even in a fiber-based platform ${ }^{55}$ or use gases as the gain material ${ }^{59}$. A similar effort can be also carried out using Er-doped and Yb-doped random fiber lasers, where the same principals will apply and similar behavior will be expected.

\section{CONCLUSIONS}

The flexible fiber-based random laser demonstrated in this paper clearly operates in the regime of Anderson localization leading to a spectrally stable and highly directional random laser. Disorder-induced localized states form several isolated channels compactly located across the transverse dimension of g-ALOF. On excitation of one of these channels by a narrow input pump, the system starts lasing by the feedback provided through the $4 \%$ reflections at each air-fiber interface. In this implementation, a point to point correspondence between the transverse position of pump and output laser is achieved. Transversely localized laser signal is associated with a more stable frequency response as long as the localization properties are unchanged. The stability of the laser spectrum is attributed to the strong mode confinement provided by the localized states in g-ALOF.

\section{CONFLICT OF INTEREST}

The authors declare no conflict of interest.

\section{AUTHOR CONTRIBUTIONS}

$\mathrm{BA}$ and $\mathrm{AM}$ wrote the manuscript and all authors contributed to its final editing; JB and TH fabricated the g-ALOF; BA and EM conducted all experiments; BA analyzed the experimental data, prepared the figures and carried out all the numerical simulations; AM conceived the original idea of making an ALOF; BA, EM, SK and AM conceived the idea of using ALOF to make a random laser, and AM led the project and supervised all aspects of the work.

\section{ACKNOWLEDGEMENTS}

We are thankful to the UNM Center for Advanced Research Computing, especially Dr Ryan Johnson, for providing access to computational resources.

1 Cao H. Random lasers: development, features and applications. Opt Photon News 2005; 16: 24-29.

2 Wiersma DS. The physics and applications of random lasers. Nat Phys 2008; 4: 359-367.

3 Graydon O. Random thoughts. Nat Photon 2013; 7: 164-165.

4 Lawandy NM, Balachandran RM, Gomes ASL, Sauvain E. Laser action in strongly scattering media. Nature 1994; 368: 436-438.

5 Wiersma DS, van Albada MP, Lagendijk A. Random laser? Nature 1995; 373 : 203-204.

6 Lawandy NM, Balachandran RM. Random laser? Nature 1995; 373: 204.

7 Wiersma DS, Lagendijk A. Light diffusion with gain and random lasers. Phys Rev E 1996; 54: 4256-4265.

8 Cao H, Zhao YG, Ho ST, Seelig EW, Wang QH et al. Random laser action in semiconductor powder. Phys Rev Lett 1999; 82: 2278-2281.

$9 \mathrm{Cao} \mathrm{H}, \mathrm{Xu}$ JY, Chang S-H, Ho ST. Transition from amplified spontaneous emission to laser action in strongly scattering media. Phys Rev E 2000; 61: 1985-1989.

10 Cao H, Xu JY, Zhang DZ, Chang S-H, Ho ST et al. Spatial confinement of laser light in active random media. Phys Rev Lett 2000; 84: 5584-5587.

11 Cao H, Ling Y, Xu JY, Cao CQ, Kumar P. Photon statistics of random lasers with resonant feedback. Phys Rev Lett 2001; 86: 4524-4527.

$12 \mathrm{Cao} \mathrm{H}$, Xu JY, Ling Y, Burin AL, Seeling EW et al. Random lasers with coherent feedback. IEEE J Sel Top Quantum Electron 2003; 9: 111-119.
13 Cao $\mathrm{H}$, Jiang $\mathrm{XY}$, Ling $\mathrm{Y}, \mathrm{Xu} \mathrm{JY}$, Soukoulis $\mathrm{CM}$. Mode repulsion and mode coupling in random lasers. Phys Rev B 2003; 67: 161101.

14 Pradhan $\mathrm{P}$, Kumar N. Localization of light in coherently amplifying random media. Phys Rev B 1994; 50: 9644-9647.

15 Jiang XY, Soukoulis CM. Time dependent theory for random lasers. Phys Rev Lett 2000; 85: 70-73.

16 Vanneste C, Sebbah P. Selective excitation of localized modes in active random media. Phys Rev Lett 2001; 87: 183903

17 Polson RC, Chipouline A, Vardeny ZV. Random lasing in $\pi$-conjugated films and infiltrated opals. Adv Mater 2001; 13: 760-764.

18 Chabanov AA, Zhang ZQ, Genack AZ. Breakdown of diffusion in dynamics of extended waves in mesoscopic media. Phys Rev Lett 2003; 90: 203903.

19 Mujumdar S, Ricci M, Torre R, Wiersma DS. Amplified extended modes in random lasers. Phys Rev Lett 2004; 93: 053903.

20 Vanneste $\mathrm{C}$, Sebbah P, Cao H. Lasing with resonant feedback in weakly scattering random systems. Phys Rev Lett 2007; 98: 143902.

21 Van der Molen KL, Tjerkstra RW, Mosk AP, Lagendijk A. Spatial extent of random laser modes. Phys Rev Lett 2007; 98: 143901.

22 Türeci HE, Ge L, Rotter S, Stone AD. Strong interactions in multimode random lasers. Science 2008; 320: 643-646.

23 Wiersma DS. Disordered photonics. Nat Photon 2013; 7: 188-196.

24 Fallert J, Dietz RJB, Sartor J, Schneider D, Klingshirn C et al. Co-existence of strongly and weakly localized random laser modes. Nat Photon 2009; 3: 279-282.

25 Fujii G. Study on low-threshold random lasing by means of finite element method. PhD thesis, Nagoya University, Chikusa-ku, Nagoya 2011.

26 Stano $P$, Jacquod $P$. Suppression of interactions in multimode random lasers in the Anderson localized regime. Nat Photon 2013; 7: 66-71.

27 Abrahams E, Anderson PW, Licciardello DC, Ramakrishnan TV. Scaling theory of localization: absence of quantum diffusion in two dimensions. Phys Rev Lett 1979; 42: 673-676.

28 Wiersma DS, Bartolini P, Lagendijk A, Righini R. Localization of light in a disordered medium. Nature 1997; 390: 671-673.

29 Van der Beek T, Barthelemy P, Johnson PM, Wiersma DS, Lagendijk A. Light transport through disordered layers of dense gallium arsenide submicron particles. Phys $\operatorname{Rev} B$ 2012; 85: 115401.

30 loffe AF, Regel AR. Non-crystalline amorphous, and liquid electronic semiconductors. Prog Semicond 1960; 4: 237-291.

31 Berry MV, Klein S. Transparent mirrors: rays, waves and localization. Eur J Phys 1997; 18: $222-228$

32 Chabanov AA, Stoytchev M, Genack AZ. Statistical signatures of photon localization. Nature 2000; 404: 850-853.

33 Bertolotti J, Gottardo S, Wiersma DS, Ghulinyan M, Pavesi L. Optical necklace states in Anderson localized 1D systems. Phys Rev Lett 2005; 94: 113903.

34 Schwartz T, Bartal G, Fishman S, Segev M. Transport and Anderson localization in disordered two-dimensional photonic lattices. Nature 2007; 446: 52-55.

35 Levi L, Rechtsman M, Freedman B, Schwartz T, Manela 0 et al. Disorder-enhanced transport in photonic quasicrystals. Science 2011; 332: 1541-1544.

36 Riboli F, Barthelemy P, Vignolini S, Intonti F, De Rossi A et al. Anderson localization of near-visible light in two dimensions. Opt Lett 2011; 36: 127-129.

37 Abaie B, Hosseini SR, Karbasi S, Mafi A. Modal analysis of the impact of the boundaries on transverse Anderson localization in a one-dimensional disordered optical lattice. Opt Commun 2016; 365: 208-214.

38 Abaie B, Mafi A. Scaling analysis of transverse Anderson localization in a disordered optical waveguide. Phys Rev B 2016; 94: 064201.

39 Abdullaev S, Abdullaev FK. On propagation of light in fiber bundles with random parameters. Radiofizika 1980; 23: 766-767.

40 De Raedt H, Lagendijk A, de Vries P. Transverse localization of light. Phys Rev Lett 1989; 62: 47-50.

41 Segev M, Silberberg Y, Christodoulides DN. Anderson localization of light. Nat Photon 2013; 7: 197-204.

42 Mafi A. Transverse Anderson localization of light: a tutorial. Adv Opt Photon 2015; 7: 459-515.

43 Karbasi S, Mirr CR, Gandomkar Yarandi P, Frazier RJ, Koch KW et al. Observation of transverse Anderson localization in an optical fiber. Opt Lett 2012; 37: 2304-2306.

44 Karbasi S, Mirr CR, Frazier RJ, Gandomkar Yarandi P, Koch KW et al. Detailed investigation of the impact of the fiber design parameters on the transverse Anderson localization of light in disordered optical fibers. Opt Express 2012; 20: 18692-18706.

45 Karbasi S, Koch KW, Mafi A. Image transport quality can be improved in disordered waveguides. Opt Commun 2013; 311: 72-76.

46 Karbasi S, Frazier RJ, Koch KW, Hawkins T, Ballato J et al. Image transport through a disordered optical fibre mediated by transverse Anderson localization. Nat Commun 2014; 5: 3362.

47 Karbasi S, Hawkins T, Ballato J, Koch KW, Mafi A. Transverse Anderson localization in a disordered glass optical fiber. Opt Mater Express 2012; 2: 1496-1503.

48 Schäfer FP, Schmidt W, Volze J. Organic dye solution laser. Appl Phys Lett 1966; 9 306-309.

49 Vasdekis AE, Town GE, Turnbull GA, Samuel IDW. Fluidic fibre dye lasers. Opt Express 2007: 15: 3962-3967.

50 Abaie B, Mobini E, Karbasi S, Ballato J, Mafi A. Observation of Lasing in an Anderson Localizing Optical Fiber. CLEO: QELS Fundamental Science; 5-10 June 2016; San Jose, CA, USA. Optical Society of America: San Jose, CA, USA. 2016.

51 COMSOL-Multiphysics. version 5.2. Wave Optics Module 2016. 
52 Siegman AE. How to (maybe) measure laser beam quality. DPSS (Diode Pumped Solid State) Lasers: Applications and Issues; 1 January 1998; Washington, DC, USA. Optical Society of America: Washington, DC, USA, 1998.

53 Mafi A, Moloney JV. Beam quality of photonic-crystal fibers. J Lightw Technol 2005; 23: 2267-2270.

54 Mujumdar S, Türck V, Torre R, Wiersma DS. Chaotic behavior of a random laser with static disorder. Phys Rev A 2007; 76: 033807.

55 Gerosa RM, Sudirman A, de S Menezes L, Margulis W, de Matos CJ. All-fiber high repetition rate microfluidic dye laser. Optica 2015; 2: 186-193.

56 Redding B, Choma AM, Cao H. Spatial coherence of random laser emission. Opt Lett 2011; 36: 3404-3406.

57 Hokr BH, Schmidt MS, Bixler JN, Dyer PN, Noojin GD et al. A narrow-band speckle-free light source via random Raman lasing. J Mod Opt 2016; 63: 46-49.

58 Redding B, Choma MA, Cao H. Speckle-free laser imaging using random laser illumination. Nat Photon 2012; 6: 355-359.
59 Nampoothiri AV, Jones AM, Fourcade-Dutin C, Mao CC, Dadashzadeh N et al. Hollowcore optical fiber gas lasers (HOFGLAS): a review [Invited]. Opt Mater Express 2012; 2: 948-961.

(c) (i) (2) This work is licensed under a Creative Commons AttributionNonCommercial-ShareAlike 4.0 International License. The images or other third party material in this article are included in the article's Creative Commons license, unless indicated otherwise in the credit line; if the material is not included under the Creative Commons license, users will need to obtain permission from the license holder to reproduce the material. To view a copy of this license, visit http:// creativecommons.org/licenses/by-nc-sa/4.0/

(C) The Author(s) 2017

Supplementary Information for this article can be found on the Light: Science \& Applications' website (http://www.nature.com/lsa). 